\title{
DIVERSIDAD GENÉTICA DE CARACTERÍSTICAS DEL ÁREA FOLIAR EN MAÍCES NATIVOS DE TAMAULIPAS BAJO ALTAS TEMPERATURAS
}

\author{
GENETIC DIVERSITY OF LEAF AREA TRAITS IN MAIZE LANDRACES NATIVE TO \\ TAMAULIPAS UNDER HIGH TEMPERATURES
}

\author{
Sergio Castro-Nava ${ }^{1 \star}$, César A. Reyes-Méndez ${ }^{2}$ y Alfredo J. Huerta ${ }^{3}$
}

\begin{abstract}
${ }^{1}$ Facultad de Ingeniería y Ciencias, Centro Universitario Adolfo López Mateos, Universidad Autónoma de Tamaulipas. 87149, Cd. Victoria Tam. Tel. y Fax 01 (834) 31-817-21 Ext. 2125. ${ }^{2}$ Campo Experimental Río Bravo, Instituto Nacional de Investigaciones Forestales, Agrícolas y Pecuarias (INIFAP). Km 61 Carr. MatamorosReynosa. 88900, Apartado Postal 172, Río Bravo, Tam. México. ${ }^{3}$ Department of Biology, Miami University. 45056, Oxford, Ohio. USA.
\end{abstract}

*Autor para correspondencia (scastro@uat.edu.mx)

\section{RESUMEN}

El Estado de Tamaulipas en México tiene una amplia variedad de maíces (Zea mays L.) nativos poco estudiados, con alto potencial de uso en el mejoramiento genético. El objetivo del estudio fue determinar la variabilidad genética de características relacionadas con el área foliar y su eficiencia para la producción de grano, en germoplasma nativo de Tamaulipas crecido en condiciones de alta temperatura. Se evaluaron 24 maíces nativos, dos materiales comerciales ('H-437' y 'VS-536') y dos generaciones avanzadas de híbridos, en dos localidades contrastantes de Tamaulipas (Río Bravo en el norte y Güémez en el centro). En Río Bravo se tuvieron condiciones óptimas de temperatura y humedad del suelo, mientras que en Güiémez se aseguraron altas temperaturas con una fecha de siembra tardía. Se registraron nueve variables agronómicas, de área foliar y eficiencia en la producción de grano. Los análisis de varianza evidenciaron amplia diversidad genética entre los maíces nativos para todas las variables estudiadas. De los 24 maíces nativos, $79 \%$ fueron superiores a los genotipos comerciales en rendimiento de grano, área de la hoja de la mazorca, área foliar total y eficiencia para la producción de grano. Estos maíces nativos pueden ser potenciales donadores de genes para mejorar la tolerancia a alta temperatura. El genotipo de mayor rendimiento de grano bajo condiciones de alta temperatura $\left(\geq 35^{\circ} \mathrm{C}\right)$ fue ' $\mathrm{C}-3004$ ' (71.4 g/planta). La eficiencia para la producción de grano mostró asociación negativa y significativa con las variables índice de área foliar $\left(-0.59^{\star *}\right)$, área foliar total $\left(-0.59^{\star *}\right)$, área de la hoja de la mazorca $\left(-0.43^{\star}\right)$ y número de hojas totales $\left(-0.51^{\star *}\right)$. Esto muestra la necesidad de hacer selección para mayor eficiencia fisiológica mediante la búsqueda del índice de área foliar óptimo para condiciones de alta temperatura.

Palabras clave: Zea mays, área de la hoja de la mazorca, varianza genética, heredabilidad, numero de hojas.

\section{SUMMARY}

The Mexican state of Tamaulipas has a wide variety of maize (Zea mays $\mathrm{L}$.) landraces not well studied and with high potential for genetic improvement. The aim of the study was to determine genetic variability for traits related to leaf area and efficiency for grain production in maize landraces from Tamaulipas grown under high temperature conditions. Twenty four maize landraces, two commercial materials (' $\mathrm{H}$ 437' and 'VS-536') and two improved advanced generations of hybrids were evaluated in two contrasting locations from Tamaulipas (Río Bravo in the North and Güémez in the Central part). The first location had optimum temperature and soil moisture, while in the second location high temperatures were ensured through late planting. Nine agronomic traits, leaf area and grain production efficiency were registered. The analysis of variance showed the presence of wide genetic diversity among the landraces for all the studied traits. Out of the $\mathbf{2 4}$ maize landraces, $79 \%$ were superior for grain yield, ear leaf area, total leaf area and efficiency for grain production in relation to commercial breeding materials. These landraces might be potential donors of genes to enhance tolerance to high temperature. The genotype with the highest grain yield under high temperature conditions $\left(\geq 35^{\circ} \mathrm{C}\right)$ was $\mathrm{C}-3004$ $(71.4 \mathrm{~g} /$ plant $)$. The efficiency for grain production had a significant and negative association with leaf area index $\left(-0.59^{* *}\right)$, total leaf area $\left(-0.59^{\star *}\right)$, ear leaf area $\left(-0.43^{*}\right)$ and total leaf number $\left(-0.51^{\star *}\right)$, thus indicating the need of selection for physiological efficiency by choosing optimum leaf area index for high temperature conditions.

Index words: Zea mays, ear leaf area, genetic variance, heritability, leaf number.

\section{INTRODUCCIÓN}

Se ha documentado sobre el origen del maíz (Zea mays L.) y se sitúa a México como su principal centro, con un largo periodo de domesticación de 6,000 a 10,000 años (Doebley, 2004) al que se atribuye la amplia diversidad reportada para diferentes características de la planta, así como la existencia de un gran número de poblaciones nativas para diversos usos.

En Tamaulipas, recientemente se han documentado 215 poblaciones nativas de maíz colectadas en diferentes zonas ecológicas (Castro et al., 2013). Algunas de estas poblaciones se han estudiado con objetivos diversos como tolerancia a estrés y alto rendimiento (Pecina-Martínez et al., 2009; Castro-Nava et al., 2011; Pecina et al., 2011; Pecina-Martínez et al., 2013). Algunas poblaciones muestran variabilidad genética en características como rendimiento y color de grano, tamaño y forma de mazorca, número de granos, altura de planta, asincronía floral, número de hojas y tolerancia al calor, entre otros (Pecina-Martínez et al., 2009; Pecina et al., 2011; Castro-Nava et al., 2011); y se han derivado líneas de estas poblaciones que han mostrado buena aptitud combinatoria (Pecina-Martínez et al., 2013). 
El rendimiento de grano es el producto de la acumulación de biomasa y de la porción del total de la misma que es asignada al grano. Durante muchos años este ha sido el principal criterio de selección en los programas de mejoramiento genético, pero es importante analizar los cambios en los atributos fisiológicos de la planta de maíz durante este proceso. Se considera que no ha cambiado el potencial máximo de la fotosíntesis (Tollenaar et al., 2000) y el potencial de rendimiento por planta determinado en óptimas condiciones (Tollenaar y Lee, 2002), ni la proporción del total de biomasa asignada al grano (índice de cosecha) (Duvick, 2005).

Para aumentar la capacidad de acumulación de biomasa es necesario hacer cambios cuantificables en la capacidad de intercepción de la radiación, debido a un incremento del índice de área foliar y cambios en su aprovechamiento por la posición de las hojas (Lee y Tollenaar, 2007). Otro aspecto para mejorar la acumulación de biomasa es la necesidad de mantener verde el área foliar durante más tiempo. La intercepción de la radiación está dada por el área foliar, mientras que la utilización de la misma es una función de la actividad fotosintética del dosel. El área foliar por planta se ha mantenido estable durante mucho tiempo, aunque al incrementar la densidad de población de plantas se incrementa el índice de área foliar; de esta manera se incrementa la intercepción de la radiación en el cultivo (Lee y Tollenaar, 2007).

Bajo esta perspectiva, en los programas de mejoramiento genético del maíz se le ha dado poca atención al área foliar como un carácter importante en la planta, y mucho menor atención al área foliar de la hoja de la mazorca, a pesar de que las hojas son el sitio en que se realiza la fotosíntesis y constituyen un factor que contribuye a la producción de biomasa (Lambert, 2010). Al respecto, la selección divergente para el área de la hoja de la mazorca ha permitido modificar el área foliar ubicada arriba de la hoja de la mazorca y otros rasgos agronómicos como el tamaño del grano y el área foliar total (Lambert, 2010).

Por ello se consideró conveniente estudiar en el germoplasma de maíz existente en Tamaulipas, las características como el área foliar total y el área de la hoja de la mazorca, como nuevos criterios de selección de genotipos morfológicamente más eficientes. El objetivo de esta investigación fue determinar la variabilidad genética para características relacionadas con el área foliar en germoplasma de maíz nativo de Tamaulipas para condiciones de alta temperatura y su eficiencia para la producción de grano.

\section{MATERIALES Y MÉTODOS}

Se estudiaron 28 genotipos de maíz, 24 de los cuales fue- ron maíces nativos del centro y sur de Tamaulipas reportados por Castro et al. (2013); dos generaciones avanzadas derivadas de híbridos $\left(\mathrm{F}_{2} 44 \times 35\right.$ y $\left.\mathrm{F}_{2} \mathrm{H}-437\right)$ y dos genotipos comerciales ('H-437' y 'VS-536'). Los maíces nativos representan la diversidad de agroecosistemas del área de colecta. El germoplasma fue evaluado en campo en dos ambientes contrastantes con la finalidad de identificar la variabilidad existente entre el germoplasma bajo condiciones de estrés por calor y establecer criterios de selección.

Las localidades fueron Río Bravo y Güémez en el Estado de Tamaulipas. La siembra se realizó el 14 de febrero en Río Bravo y el 21 de marzo en Güémez durante el ciclo OtoñoInvierno de 2008, esta última considerada como fecha tardía para asegurar la ocurrencia de temperaturas mayores a la óptima $\left(\geq 35^{\circ} \mathrm{C}\right)$; de la siembra a la madurez fisiológica la temperatura promedio fue de 38.2 y $18{ }^{\circ} \mathrm{C}$, máxima y mínima respectivamente, con un total de 119 a $130 \mathrm{~d}$ con temperatura $\geq 35^{\circ} \mathrm{C}$, y una precipitación de $187 \mathrm{~mm}$.

El experimento se estableció bajo un diseño experimental en bloques completos al azar con tres repeticiones. Las plantas crecieron en un surco de $5 \mathrm{~m}$ de longitud con espacios entre surcos de $0.80 \mathrm{~m}$ y entre plantas de $0.25 \mathrm{~m}$. Se fertilizó con la fórmula $120 \mathrm{~N}-40 \mathrm{P}-00 \mathrm{~K}$. Las malezas se controlaron con el paso de dos escardas y aplicación de herbicida, de acuerdo con las recomendaciones para maíz en cada ambiente. Durante el experimento se aplicaron dos riegos de auxilio, el primero cuando se detectó la iniciación floral (40 d después de la emergencia) y el segundo al inicio de la floración (70 d después de la emergencia).

Las variables medidas fueron: área foliar total $\left(\mathrm{cm}^{2}\right)$ y área de la hoja de la mazorca $\left(\mathrm{cm}^{2}\right)$, determinadas con un medidor de área foliar (LI-3000A, LI-COR; Lincoln, NE, USA) cuando las plantas alcanzaron la madurez fisiológica en el grano, y midiendo sólo las hojas fotosintéticamente activas en cinco plantas por parcela. Se cuantificó el número de hojas arriba de la mazorca y el número de hojas totales en cinco plantas por parcela, desde la emergencia de la plántula hasta la hoja bandera, con marcas de pintura blanca. Al final de la floración se calculó el índice de área foliar mediante la relación entre el área foliar total y la superficie del terreno por planta.

El rendimiento de grano $(\mathrm{g})$ por planta se midió en cinco plantas individuales por parcela y la humedad del grano se ajustó a $12 \%$. Se determinó el peso de 100 granos por parcela para estimar el peso individual del grano (g), mientras que el número de granos por mazorca se estimó con el cociente del rendimiento de grano entre el peso individual del grano. La eficiencia para la producción de grano se estimó mediante el cociente entre el rendimiento de grano y el índice de área foliar, como sugirieron Rutger et al. (1971). 
Además, se determinó la proporción del área de la hoja de la mazorca con respecto al área foliar total expresada en porcentaje.

Los datos fueron sometidos a un análisis de componentes de varianza (SAS Institute, 2010) por localidad, y se calculó la heredabilidad en sentido amplio $\left(H^{2}\right)$ para cada una de las variables en estudio. Los componentes de varianza y $H^{2}$ se determinaron con el procedimiento usado por Johnson et al. (1955) para cada una de las localidades. Los componentes obtenidos en Güémez se compararon con los obtenidos en Río Bravo, considerando el efecto de la alta temperatura (temperaturas $\geq 35^{\circ} \mathrm{C}$ ). La comparación de medias entre tratamientos se hizo con la prueba de Tukey $(\mathrm{P} \leq 0.05)$. La asociación entre variables se determinó mediante correlaciones de Pearson $(\mathrm{P} \leq 0.05)$. La relación entre el rendimiento de grano por planta y el área foliar total fue analizada con un modelo de regresión cuadrática con el PROC REG de SAS.

\section{RESULTADOS Y DISCUSIÓN}

El análisis de varianza por localidades muestra que la respuesta de los genotipos de maíz fue significativamente diferente $(\mathrm{P} \leq 0.05)$ para todas las variables (Cuadro 1), excepto para peso individual de grano en Río Bravo. Esto indica la existencia de un alto grado de variabilidad genética susceptible de ser aprovechada en un programa de mejoramiento genético. Similares resultados han sido reportados por Grzesiak (2001), Hortelano et al. (2008), Pecina-Martínez et al. (2009), Idris y Abuali (2011), Pecina et al. (2011) y Hortelano et al. (2012), para diferentes variables en maíz.

Los promedios para genotipos comerciales y nativos en cada variable y las variaciones en cada ambiente se muestran en el Cuadro 2. La diversidad mostrada por los maíces nativos en las variables en estudio indica que la selección para caracteres como área foliar total y área de la hoja de la mazorca puede ser efectiva, aunque lo que resulte estará en función de la magnitud de la heredabilidad (Johnson et al., 1955). En el ambiente con alta temperatura (Güémez), todas las variables tuvieron valores más bajos en comparación con el comportamiento manifestado en la localidad con temperatura óptima (Río Bravo).

En general, como resultado del estrés por calor el rendimiento de grano de los maíces nativos fue reducido (34 $\%$ ); así mismo se observaron reducciones en: número de granos (29\%), área foliar total (29\%), área de la hoja de la mazorca (21\%), número de hojas arriba de la mazorca (20 \%) e índice de área foliar (29\%). De los 24 maíces nativos, $67 \%$ tuvieron rendimiento de grano superior al promedio del ambiente con alta temperatura, mientras que $79 \%$ superaron a los genotipos comerciales; además, $50 \%$ de los maíces nativos de mayor rendimiento de grano tuvieron también mayor área foliar total y área de la hoja de la mazorca, y fueron los de mayor eficiencia en la producción de grano por planta.

El genotipo de mayor rendimiento de grano bajo condiciones de alta temperatura fue 'C-3004' (71.4 g/planta). El área de la hoja de la mazorca representó entre 10 a $15 \%$ del total del área foliar por planta (Cuadro 2) en condiciones de alta temperatura, y de 9 a $14 \%$ en condiciones óptimas; esto es relevante porque las láminas foliares son el sitio principal donde se lleva a cabo el proceso de fotosíntesis, un factor importante que contribuye a la producción de biomasa y, por tanto, a la producción de grano (Lambert, 2010). Por ello es que algunos de estos maíces nativos ('C-3004' y 'C3009 ') podrían ser considerados como base para su aprovechamiento en un programa para su mejoramiento genético en el que se utilice al área de la hoja de la mazorca como

Cuadro 1. Cuadrados medios (CM) y coeficientes de variación (CV) del análisis de varianza por localidad de nueve variables evaluadas en 28 genotipos de maíz de Tamaulipas, México.

\begin{tabular}{lccccc}
\hline \multirow{2}{*}{ Variable } & \multicolumn{2}{c}{ Río Bravo } & & \multicolumn{2}{c}{ Güémez } \\
\cline { 2 - 3 } \cline { 5 - 6 } & $\mathrm{CM}$ & $\mathrm{CV}(\%)$ & & $\mathrm{CM}$ & $\mathrm{CV}(\%)$ \\
\hline Área foliar total $\left(\mathrm{cm}^{2}\right)$ & $1,526,827^{* * *}$ & 13.5 & & $1,258,977^{* * *}$ & 14.7 \\
Área de la hoja de la mazorca $\left(\mathrm{cm}^{2}\right)$ & $11,955^{*}$ & 11.4 & & $6749^{*}$ & 13.8 \\
Número de hojas arriba de la mazorca & $0.70^{* * *}$ & 8.4 & & $0.53^{* *}$ & 8.0 \\
Número de hojas totales & $5.42^{* * *}$ & 4.8 & & $2.97^{* * *}$ & 6.1 \\
Índice de área foliar & $0.46^{* * *}$ & 14.5 & & $0.314^{* * *}$ & 14.7 \\
Rendimiento de grano por planta $(\mathrm{g})$ & $400^{* * *}$ & 16.9 & & $581^{* * *}$ & 30.9 \\
Eficiencia para la producción de grano & $140^{* * *}$ & 25.2 & & $281^{* * *}$ & 35.2 \\
Número de granos por mazorca & $8536^{* * *}$ & 15.2 & & $9328^{* * *}$ & 24.7 \\
Peso individual del grano $(\mathrm{g})$ & $0.002 \mathrm{~ns}$ & 15.0 & & $0.002^{*}$ & 18.3 \\
\hline
\end{tabular}

ns, ${ }^{*}, * *, * *$ : no significativo $\mathrm{P} \geq 0.05$, significativo a $\mathrm{P} \leq 0.05, \mathrm{P} \leq 0.01$ y $\mathrm{P} \leq 0.001$, respectivamente. 
Cuadro 2. Variación fenotípica en diferentes caracteres de maíces nativos de Tamaulipas y promedios de maíces nativos y comerciales en dos ambientes del Estado de Tamaulipas, México.

\begin{tabular}{|c|c|c|c|c|c|c|}
\hline \multirow[b]{2}{*}{ Variable } & \multicolumn{3}{|c|}{ Río Bravo } & \multicolumn{3}{|c|}{ Güémez } \\
\hline & Variación & $\begin{array}{l}\text { Promedio } \\
\text { comerciales }\end{array}$ & $\begin{array}{c}\text { Promedio } \\
\text { nativos }\end{array}$ & Variación & $\begin{array}{c}\text { Promedio } \\
\text { comerciales }\end{array}$ & $\begin{array}{c}\text { Promedio } \\
\text { nativos }\end{array}$ \\
\hline Área foliar total $\left(\mathrm{cm}^{2}\right)$ & $3536-6286$ & 4730.7 & 4906.0 & 2204-4539 & 2988.3 & 3464.8 \\
\hline Área de la hoja de la mazorca $\left(\mathrm{cm}^{2}\right)$ & $434-785$ & 579.3 & 544.9 & $340-508$ & 401.5 & 430.6 \\
\hline Número de hojas arriba de la mazorca & $5.3-7.4$ & 7.1 & 6.1 & $4.3-5.8$ & 5.4 & 4.9 \\
\hline Número de hojas totales & $17-23$ & 20.3 & 19.8 & $15-21$ & 18.6 & 18.7 \\
\hline Índice de área foliar & $1.25-3.14$ & 2.0 & 2.45 & $1.10-2.27$ & 1.49 & 1.73 \\
\hline Rendimiento de grano por planta (g) & $27-91$ & 59.1 & 68.4 & $16-71$ & 36.9 & 45.2 \\
\hline Eficiencia para la producción de grano & $8.9-42.1$ & 28.9 & 29.0 & $7.6-46.4$ & 24.9 & 27.0 \\
\hline Número de granos por mazorca & $131-412$ & 319.2 & 321.4 & $107-318$ & 190.2 & 227.8 \\
\hline Peso individual del grano (g) & $0.17-0.28$ & 0.18 & 0.22 & $0.14-0.24$ & 0.20 & 0.20 \\
\hline Área hoja de la mazorca/área foliar total (\%) & $8.8-14$ & 12.5 & 11.2 & $9.6-14.6$ & 13.4 & 12.7 \\
\hline
\end{tabular}

criterio de selección.

La heredabilidad estimada para las diferentes características en condiciones de alta temperatura varió de 10 a 27 $\%$ (Cuadro 3). Los valores de heredabilidad para todos los caracteres fueron bajos, debido a que las varianzas fenotípicas fueron altas, como resultado de la interacción de los genotipos y el ambiente; además, la heredabilidad se mantuvo sin cambio bajo condiciones de alta temperatura (Güémez) en relación a la condición óptima (Río Bravo). La varianza genética en ambas localidades no fue superior a $33 \%$ en relación a la varianza fenotípica.

Como se esperaba, el coeficiente de variación fenotípica (Cuadro 3) fue más alto que el coeficiente de variación genética para todos los caracteres, por efecto del ambiente sobre los genotipos. Resultados similares fueron encontrados en maíz por Idris y Abuali (2011) y en trigo (Triticum aestivum (L.) Thell.) por Ehdaie y Waines (1989), Espitia et al. (2004) y Akçura (2009). Los coeficientes de variación genética máximos en el ambiente de alta temperatura correspondieron a las variables rendimiento de grano por planta y la eficiencia para la producción de grano. Esto sugiere la oportunidad de aplicar selección para estas variables y aprovechar la amplia variabilidad observada en condiciones de alta temperatura. Los coeficientes de variación genética para índice de área foliar y área foliar total fueron intermedios, los cuales permiten todavía efectuar selección.

Para incrementar la eficiencia biológica de las plantas es necesario conocer algunas características relacionadas con el área foliar, como lo señalaron Rutger et al. (1971), y en el caso del maíz no existe mucha investigación sobre los cambios en el área foliar como resultado del mejoramiento genético (Lambert, 2010). Incrementar el área foliar puede resultar en un incremento en el rendimiento de grano, sin alterar el periodo de llenado de grano (Schoper et al., 1982).

Las correlaciones fenotípicas entre las variables estudiadas en el ambiente con alta temperatura indican que hubo una asociación significativa y positiva del área foliar total con el área de la hoja de la mazorca $\left(0.83^{* *}\right)$, con el índice de área foliar $\left(0.98^{\star *}\right)$ y con el número de hojas totales $\left(0.54^{* *}\right)$; mientras que el área de la hoja de la mazorca estuvo asociada con el índice de área foliar $\left(0.83^{\star *}\right)$ y con el número de hojas totales $\left(0.51^{\star *}\right)$. Además, el número de hojas totales presentó una asociación positiva y significativa con el índice de área foliar $\left(0.53^{\star *}\right)$. La eficiencia para la producción de grano estuvo asociada positiva y significativamente con el número de granos $\left(0.87^{\star *}\right)$ y el peso individual del grano $\left(0.54^{\star \star}\right)$.

Como se esperaba, el rendimiento de grano estuvo asociado positiva y significativamente con la eficiencia para la producción de grano $\left(0.89^{* *}\right)$, con el número de granos $\left(0.92^{\star *}\right)$ y el peso individual del grano $\left(0.70^{\star *}\right)$. Una observación interesante fue la asociación negativa y significativa de la eficiencia para la producción de grano con el índice de área foliar $\left(-0.59^{* *}\right)$, con el área foliar total $\left(-0.59^{* *}\right)$, con el área de la hoja de la mazorca $\left(-0.43^{\star}\right)$ y con el número de hojas totales $\left(-0.51^{\star *}\right)$.

Esto indica la necesidad de practicar selección para mayor eficiencia fisiológica en la producción de grano, mediante la búsqueda de la mayor área foliar por planta (Figura 1), y del índice de área foliar óptimo para la tolerancia al estrés por alta temperatura, ya que como señalaron Tollenaar y Lee (2006), el área foliar óptima y el índice de cosecha son 
Cuadro 3. Componentes de varianza y heredabilidad $\left(H^{2}\right)$ estimados en diferentes variables de germoplasma de maíz nativo en dos ambientes contrastantes del estado de Tamaulipas, México.

\begin{tabular}{|c|c|c|c|c|c|c|c|c|}
\hline \multirow{2}{*}{ Variable } & \multicolumn{4}{|c|}{ Río Bravo } & \multicolumn{4}{|c|}{ Güémez } \\
\hline & CVG & $\mathrm{CVF}$ & $\sigma_{G}^{2}$ & $H^{2}(\%)$ & CVG & $\mathrm{CVF}$ & $\sigma_{G}^{2}$ & $H^{2}(\%)$ \\
\hline Área foliar total $\left(\mathrm{cm}^{2}\right)$ & 12.3 & 25.3 & 361,814 & 23.7 & 16.9 & 32.7 & 335,175 & 26.6 \\
\hline Área de la hoja de la mazorca $\left(\mathrm{cm}^{2}\right)$ & 9.48 & 20.0 & 2691 & 22.5 & 7.6 & 19.2 & 1083 & 16.1 \\
\hline Número de hojas arriba de la mazorca & 6.1 & 13.5 & 0.143 & 20.5 & 7.2 & 14.8 & 0.12 & 23.4 \\
\hline Número de hojas totales & 6.2 & 11.7 & 1.501 & 27.7 & 4.0 & 9.2 & 0.548 & 18.5 \\
\hline Índice de área foliar & 13.9 & 28.1 & 0.11 & 24.4 & 16.9 & 32.8 & 0.08 & 26.6 \\
\hline Rendimiento de grano por planta (g) & 13.7 & 29.1 & 88.7 & 22.2 & 25.6 & 54.1 & 130 & 22.4 \\
\hline Eficiencia para la producción de grano & 18.4 & 40.8 & 28.4 & 20.3 & 29.3 & 62.1 & 62.6 & 22.3 \\
\hline Número de granos por mazorca & 14.1 & 28.8 & 2054 & 24.1 & 20.3 & 42.9 & 2077 & 22.3 \\
\hline Peso individual del grano (g) & 10.7 & 18.4 & 0.0005 & 33.3 & 7.1 & 22.4 & 0.0002 & 10.0 \\
\hline
\end{tabular}

$\mathrm{CVG}=$ coeficiente de variación genética; $\mathrm{CVF}$ = coeficiente de variación fenotípica; $\sigma_{G}^{2}=$ varianza genética; $H^{2}=$ heredabilidad en sentido amplio.

dos características que influyen en el aprovechamiento de la variación y la heterosis. La estrategia más adecuada para aprovechar la variación observada es establecer la densidad de población óptima para cada población, como también señalaron Lee y Tollenaar (2007), y además es necesario considerar la formación de un arquetipo específico para aprovechar el potencial genético del rendimiento (Rasmusson, 1987). Tollenaar y Lee (2002) sugirieron que para incrementar el rendimiento de grano es necesario considerar, además de la aplicación de metodologías de mejoramiento genético, la respuesta de interacción entre el genotipo y las prácticas agronómicas.

Aunque la contribución individual de las hojas en la planta, así como el número de hojas arriba y abajo de la mazorca al rendimiento de grano y la acumulación de biomasa, no están suficientemente estudiadas (Subedi y Ma, 2005), es importante considerar que las hojas arriba de la mazorca y la misma hoja de la mazorca son las más jóvenes en la planta, por lo que tienen las tasas fotosintéticas mayores y se mantienen por más tiempo (Thiagarajah et al., 1981), y son también las que aportan en mayor proporción los fotoasimilados a la mazorca durante el llenado de grano (Tollenaar, 1977), lo cual no ocurre con las hojas posicionadas abajo de la mazorca, por ser las primeras en aparecer y las más sombreadas (Dwyer y Stewart, 1986).

Pocas evidencias existen en la literatura en relación con la selección para magnitud del área foliar de la hoja de la mazorca; sin embargo, Lambert (2010) señaló que este criterio de selección se ha reflejado en modificar caracteres como el área foliar arriba de la mazorca. Aunque la selección para una reducida área foliar posicionada arriba de la mazorca resultó en una reducción de la longitud y anchura de sus hojas, también dio un incremento del rendimiento de gra- no (Fischer et al., 1987), tal vez porque ello permitió mejor iluminación de las hojas inferiores.

\section{CONCLUSIONES}

Existe una amplia variabilidad genética entre los maíces nativos de Tamaulipas en las variables directamente relacionadas con el área foliar y la eficiencia para la producción de grano en condiciones de alta temperatura. Los maíces nativos de mayor rendimiento de grano tuvieron la mayor área foliar total y la mayor área de la hoja de la mazorca, y fueron los de mayor eficiencia para la producción de grano. De los 24 maíces nativos, 79 \% fueron superiores al promedio en rendimiento de grano, área de la hoja de la mazorca, área foliar total y eficiencia para la producción de grano, en relación con los genotipos mejorados comerciales.

\section{AGRADECIMIENTOS}

Los autores agradecen al Consejo Nacional de Ciencia y Tecnología (CONACyT) el financiamiento para la realización de esta investigación a través del proyecto SEP 2003-CO2-44713.

\section{BIBLIOGRAFÍA}

Akçura M. (2009) Genetic variability and interrelationship among grain yield and some quality traits in Turkish winter durum wheat landraces. Turkish Journal Agriculture and Forestry 33:547-556.

Castro-Nava S., V. H. Ramos-Ortíz, C. A. Reyes-Méndez, F. BrionesEncinia y J. A. López-Santillán (2011) Preliminary field screening of maize landrace germplasm from northeastern México under high temperatures. Maydica 56:77-82.

Castro S., J. A. López, J. A. Pecina, M. C. Mendoza y C. A. Reyes (2013) Exploración de germoplasma nativo de maíz en el centro y sur de Tamaulipas, México. Revista Mexicana de Ciencias Agrícolas 4:645-653.

Doebley J. (2004) The genetics of maize evolution. Annual Review of Genetics 38:37-59.

Duvick D. N. (2005) The contribution of breeding to yield advances in 


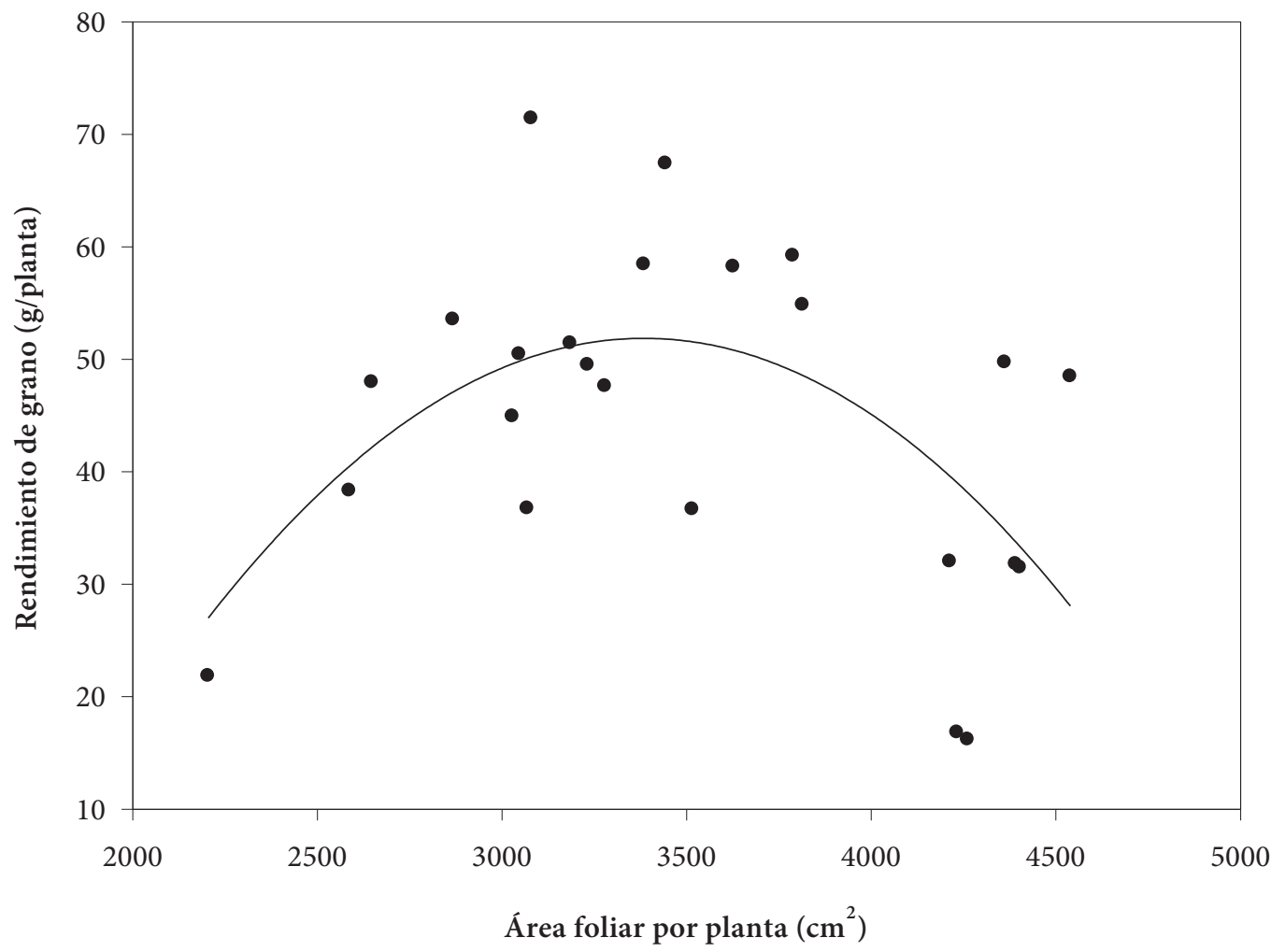

Figura 1. Rendimiento de grano por planta en función del área foliar por planta en 24 maíces nativos de Tamaulipas bajo condiciones de alta temperatura, ajustado con un modelo de regresión cuadrático $\left(Y=-1.79 E-05 X^{2}+0.1307 X-167.99 ; R^{2}=\right.$ $0.37 ; \mathrm{P}=0.008)$.

maize (Zea mays L.). Advances in Agronomy 86:83-145.

Dwyer L. M. and D. W. Stewart (1986) Effect of leaf age and position on net photosynthetic rates in maize (Zea mays L.). Agricultural and Forest Meteorology 37:29-46.

Ehdaie B. and J. G. Waines (1989) Genetic variation, heritability and path-analysis in landraces of bread wheat from southwestern Iran. Euphytica 41:183-190.

Espitia E., H. E. Villaseñor, R. J. Peña, J. Huerta y A. Limón (2004) Calidad industrial de trigos harineros mexicanos para temporal. II. Variabilidad genética y criterios de selección. Revista Fitotecnia Mexicana 27:41-47.

Fischer K. S., G. O. Edmeades and E. C. Johnson (1987) Recurrent selection for reduced tassel branch number and reduced leaf area density above the ear in tropical maize populations. Crop Science 27:1150-1156.

Grzesiak S. (2001) Genotypic variation between maize (Zea mays L.) single cross hybrids in response to drought stress. Acta Physiologiae Plantarum 23:443-456.

Hortelano R., A. Gil, A. Santacruz, S. Miranda y L. Córdova (2008) Diversidad morfológica de maíces nativos del Valle de Puebla. Agricultura Técnica en México 34:189-200.

Hortelano R., A. Gil, A. Santacruz, H. López, P. A. López y S. Miranda (2012) Diversidad fenotípica de maíces nativos del altiplano centro-oriente del estado de Puebla, México. Revista Fitotecnia Mexicana 35:97-109.

Idris A. E. and A. I. Abuali (2011) Genetic variability for vegetative and yield traits in maize (Zea mays L.) genotypes. International Research Journal of Agricultural Science and Soil Science 1:408-411.

Johnson H. W., H. F. Robinson and R. E. Comstock (1955) Estimates of genetic and environmental variability in soybeans. Agronomy Journal 47:314-318.

Lambert R. J. (2010) Divergent selection for ear leaf area in maize. Maydica 55:155-161.
Lee E. A. and M. Tollenaar (2007) Physiological basis of successful breeding strategies for maize grain yield. Crop Science 47(S3):S202S215.

Pecina-Martínez J. A., M. C. Mendoza-Castillo, J. A. López-Santillán, F. Castillo-González y M. Mendoza-Rodríguez (2009) Respuesta morfológica y fenológica de maíces nativos de Tamaulipas a ambientes contrastantes de México. Agrociencia 43:681-694.

Pecina J. A., M. C. Mendoza, J. A. López, F. Castillo, M. Mendoza y J. Ortiz (2011) Rendimiento de grano y sus componentes en maíces nativos de Tamaulipas evaluados en ambientes contrastantes. Revista Fitotecnia Mexicana 34:85-92.

Pecina-Martínez J. A., M. C. Mendoza-Castillo, F. Castillo-González, J. A. López-Santillán, M. Mendoza-Rodríguez and C. A. Reyes-Méndez (2013) Genetic potential of $S_{1}$ lines derived from native maize populations of Tamaulipas, México. Maydica 58:127-134.

Rasmusson D. C. (1987) An evaluation of ideotype breeding. Crop Science 27:1140-1146.

Rutger J. N., C. A. Francis and C. O. Grogan (1971) Diallel analysis of ear leaf characteristics in maize (Zea mays L.). Crop Science 11:194-195.

SAS Institute (2010) SAS/STAT User's guide: Version 9.2 SAS Institute, Inc. Cary, North Carolina, USA. 1689 p.

Schoper J. B., R. R. Johnson and R. J. Lambert (1982) Maize yield response to increased assimilate supply. Crop Science 22:11841189.

Subedi K. D. and B. L. Ma (2005) Ear position, leaf area, and contribution of individual leaves to grain yield in conventional and leafy maize hybrids. Crop Science 45:2246-2257.

Thiagarajah M. R., L. A. Hunt and J. D. Mahon (1981) Effects of position and age on leaf photosynthesis in corn (Zea mays L.). Canadian Journal of Botany 59:28-33.

Tollenaar M. (1977) Sink-source relationships during reproductive 
development in maize: a review. Maydica 22:49-75.

Tollenaar M., J. Ying and D. N. Duvick (2000) Genetic gain in corn hybrids from the Northern and Central Corn Belt. Proceedings of the $55^{\text {th }}$ Annual Corn and Sorghum Research Conference Chicago Ill. 5-8 December 2000. American Seed Trade Association. Washington, D C. pp:53-62.
Tollenaar M. and E. A. Lee (2002) Yield potential, yield stability and stress tolerance in maize. Field Crops Research 75:161-170.

Tollenaar M. and E. A. Lee (2006) Dissection of physiological processes underlying grain yield in maize by examining genetic improvement and heterosis. Maydica 51:399-408. 